Proceedings of the 18th National Conference on Superconductivity, Krynica Morska, Poland, October 8-13, 2017

\title{
Microavalanches Size Distribution in Fine Structure of Thermomagnetic Flux Jumps in the $\mathrm{V}_{3}$ Si Monocrystal Superconductor
}

\author{
O.I. KuchuK ${ }^{a}$, V.F. Rusakov ${ }^{b}$, A. NabialeK ${ }^{c}$, O.M. ChumaK $^{a, c}$, M.V. Zalutskit $^{d}$ \\ AND V.V. ChaBANENKO ${ }^{a, *}$ \\ ${ }^{a}$ O.O. Galkin Donetsk Institute for Physics and Engineering, N.A.S., Pr. Nauki, 46, 03028 Kyiv, Ukraine \\ ${ }^{b}$ Vasyl' Stus Donetsk National University, Ul. 600-letiia 21, 21021 Vinnitsa, Ukraine \\ ${ }^{c}$ Institute of Physics, Polish Academy of Sciences, Aleja Lotnikow 32/46, PL-02668 Warsaw, Poland \\ ${ }^{d}$ G.V. Kurdyumov Institute for Metal Physics of the N.A.S., Ac. Vernadsky Bld 36, 03142 Kyiv, Ukraine
}

The fine structure of huge thermomagnetic flux jumps in $\mathrm{V}_{3} \mathrm{Si}$ single crystal was analyzed in detail. Structure of avalanches consists of several hundreds of microavalanches. The magnitude of the flux of the microavalanches and their duration were determined. The histograms of the distribution of microflux jumps magnitudes are plotted (the number of microavalanches as a function of the outgoing flux size) for different temperatures. The role of microstructure pinning centers was discussed on the assumption that microavalanches are jumps of flux bundles. In such case, fine structure of the avalanche can characterize the parameters of the pinning centers (size, number of pinned vortices, etc.).

DOI: 10.12693/APhysPolA.135.41

PACS/topics: 74.70.Ad, 74.25.Sv

\section{Introduction}

It is known [1] that when a hard type II superconductor is placed in the external magnetic field, at certain conditions, the thermomagnetic avalanches may occur, which manifest themselves as giant jumps of magnetic flux $\Delta \Phi_{a v}(t), t$ is time. They may occur in cases increasing (screening mode) and decreasing (trapping mode) external magnetic field.

In previous work [2], using the data acquisition board $(\mathrm{DAQ})$ with the time resolution to within $\approx 10^{-7} \mathrm{~s}$, we reveal fine structure of giant flux jumps going out from $\mathrm{V}_{3} \mathrm{Si}$ single crystal (flux trapping mode) as a result of thermomagnetic avalanche during slow ramp of external magnetic field. It was shown that avalanches consist of a large number of very short $(\approx 10 \mu \mathrm{s})$ microavalanches and that each of the microavalanches has its own structure. The originating of fine structure is connected with discrete structure of pinning centers. Earlier [3-10] the role of microstructure pinning centers in slow dynamic of flux was discussed on the assumption that microavalanches are jumps of flux bundles. Therefore, methodically, fine structure of the avalanches may be a new additional facility for parameter of pinning centers (size, number of pinned vortices, etc.) investigation.

In present paper the magnitude of the flux in the microavalanches $\Delta \Phi_{\mu a v}$ and its duration at different temperatures were analyzed. The histograms of the distribution of microavalanches magnitudes (the number of

*corresponding author; e-mail: vikchabanenko@gmail.com avalanches as a function of the size of the leaving flux) are plotted. One of the physical mechanisms of microstructure pinning centers appearing in $\mathrm{V}_{3} \mathrm{Si}$ crystal was considered.

The dynamics of the flux at thermomagnetic avalanches in single crystal also was considered in the framework of the model of magnetic and thermal diffusion based on the approach developed in Refs. [1, 11, 12]. An analysis of this process for conventional superconductor $\mathrm{V}_{3} \mathrm{Si}$ was performed in locally adiabatic approximation, for which the thermal diffusivity, $D_{t h}$, of the investigated sample is assumed to be significantly lower than the magnetic diffusivity, $D_{m}\left(D_{t h} \ll D_{m}\right)$. Such conditions usually occur in conventional superconductors [1]. The thermal diffusivity is determined by $D_{t h}=\kappa / C$ where $\kappa$ is the thermal conductivity and $C$ is the specific heat. The magnetic diffusivity can be expressed as $D_{m}=\rho_{f m} / \mu_{0}$, where $\rho_{f m}$ is the electrical resistance determined by the motion of the magnetic flux (for example, flux creep, flux flow). The values of magnetic diffusivity at the initial and at final stages of the thermomagnetic avalanches were estimated from experimental data. These values of the magnetic diffusivity are lower than that typical for the normal state of conventional superconductor.

\section{Experiment}

All experiments were performed in $12 \mathrm{~T}$ superconducting magnet with variable temperature insert. A pick-up coil (6 turns) was wound around the investigated sample (see Fig. 1a, $\mathrm{V}_{3} \mathrm{Si}$ disc with diameter $8.5 \mathrm{~mm}$ and thickness $0.85 \mathrm{~mm}$, the critical temperature is $T_{c}=16.8 \mathrm{~K}$ ). 
The resistance ratio $R_{300 K} / R_{20} \mathrm{~K}$ of our single crystal was measured to be 12 ; specific resistance of the sample at the temperature of liquid nitrogen was $\rho_{78} K=$ $20 \mu \Omega \mathrm{cm}$ ). For impedance measurement (Fig. 1b) of the sample HP bridge was used. We registered time dependence of the coil voltage $U(t) \sim \mathrm{d} \Phi / \mathrm{d} t$ during the following flux jumps with the aid of DAQ (the time resolution to within $\approx 10^{-7} \mathrm{~s}$ ) in computer. The disk magnetization $M$ was obtained as the differential signal from the two Hall probes. One of the probes was put on the surface of the investigated sample in the center of the disc and registered the local magnetic field $B_{\text {surf }}$ (Fig. 1a). The second probe measured the external magnetic field $\mu_{0} H_{\text {ext }}$.

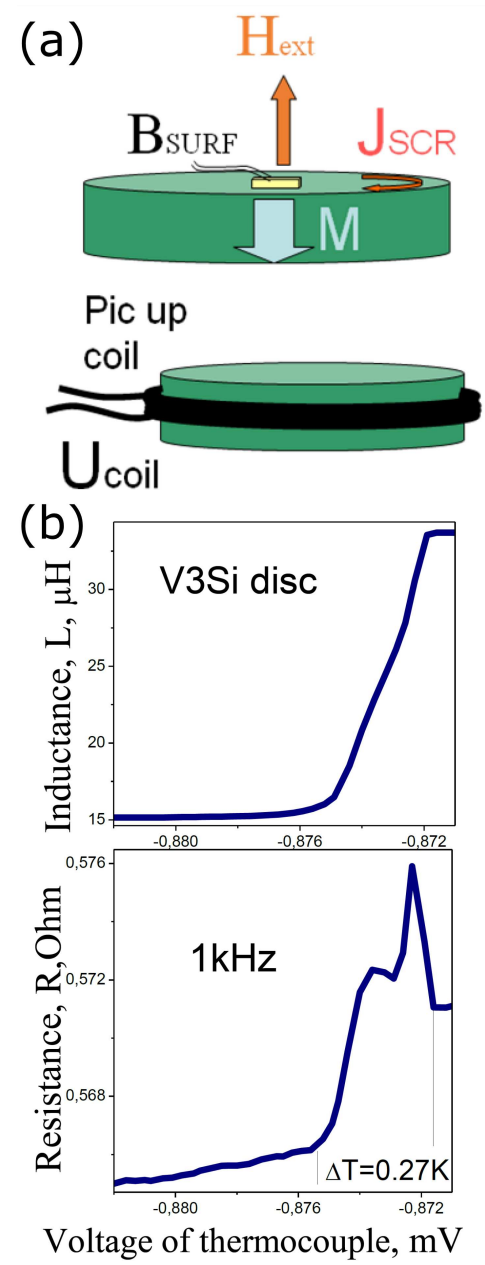

Fig. 1. (a) Sample geometry; Hall probe at the surface of $\mathrm{V}_{3} \mathrm{Si}$ disc for local magnetic induction $\left(B_{\text {surf }}\right)$ measurement; $J_{\mathrm{SCR}}$ - screening current; pick up coil show lower. (b) The superconducting transition in impedance (inductance and resistance) of sample at frequency $1 \mathrm{kHz}$.

The magnetic field was directed perpendicular to the surface of the disk and parallel to the crystallographic orientation of the crystal (111). A more detailed description of the experiment is presented in [2], the details of crystals preparation and the magnetic properties of this sample - see in [13].

\section{Results}

In Fig. 2 the magnetization hysteresis loop $M\left(H_{\text {ext }}\right)$ recorded at a temperature of $4.2 \mathrm{~K}$ is shown. Jumps of the magnetic flux, both in increasing field (screening mode, first (Q1) and third (Q3) quadrant) and in decreasing field (trapping mode, second (Q2) and fourth (Q4) quadrant) are represented on this loop. The insets in Fig. 2 show the voltage signals for the avalanches on the coil, calculated per one turn of the coil, in three quadrants. One can see that microflux jumps appear as a set of irregularly shape voltage impulses superimposed on an induced smoothly varying voltage due to the flux exit, but only for the flux trapping mode (quadrants Q2 and Q4). The magnitude of the flux $\Delta \Phi_{a v}$ in each of the three quadrants (see inset frames in Fig. 2) is approximately of $20 \mu \mathrm{Wb} \approx 10^{10} \Phi_{0}$.

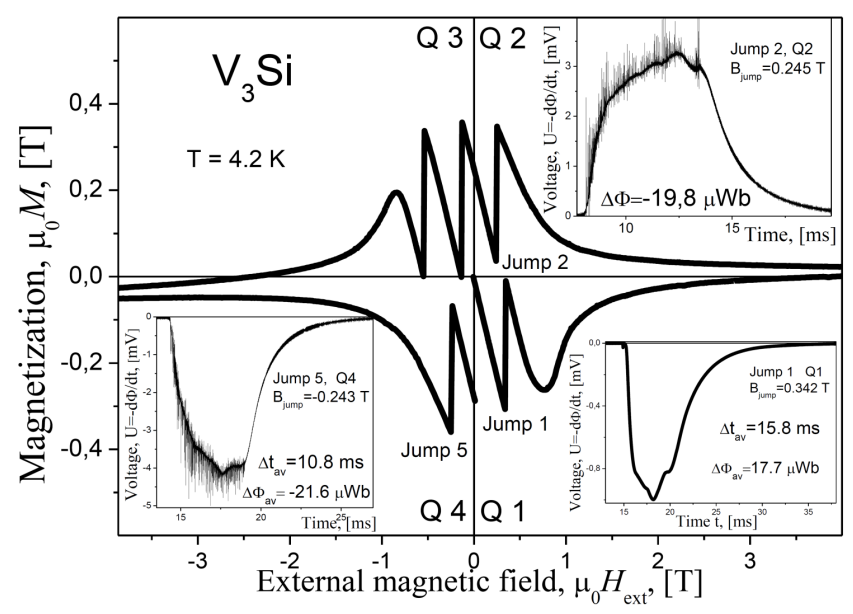

Fig. 2. Main frame: hysteresis loop of magnetization $M\left(H_{\text {ext }}\right)$; inset frames: time dependence of the voltage $(U)$ per one turn of the coil, registered during the flux jump $\left(\Delta \Phi_{a v}\right)$ in different quadrants Q1, Q2, and Q4; $B_{\text {jump }}$ is the field of flux jump; $\Delta t_{a v}$ is the duration of flux avalanche; $T=4.2 \mathrm{~K}$.

The absence of a fine structure in the first quadrant can have the following explanation. When the magnetic flux enters the sample in the screening mode, all avalanches are generated at the sample boundary and, under the pressure of an external magnetic field, individual vortices penetrate into its volume, with subsequent redistribution and fixation at the pinning centers. The magnetic flux has a quantum character determined by the quantum of the flux, which carries a single vortex, but it cannot be fixed at the sensitivity level of the used equipment. Further, the flux that has already entered the sample, even moving discretely (bundles of vortices) through the pinning centers inside the superconductor, is not registered by the coil, since the amount of flux inside the sample does not change. Therefore, the incoming avalanches do not exhibit a microstructure.

When the flux goes out the sample, the picture changes. The trapped flux is fixed at the pinning centers, which can have different sizes and different nature, 
and consequently the force of interaction with the vortices will be different. These differences will lead to the fact that the vortices will jump from the pinning centers not simultaneously. This discreteness, in turn, leads to microstructure of the outgoing avalanches. It should be emphasized that a fine structure arises as a result of the jumping of the vortex bundles from the pinning centers located at the outgoing flux front line.
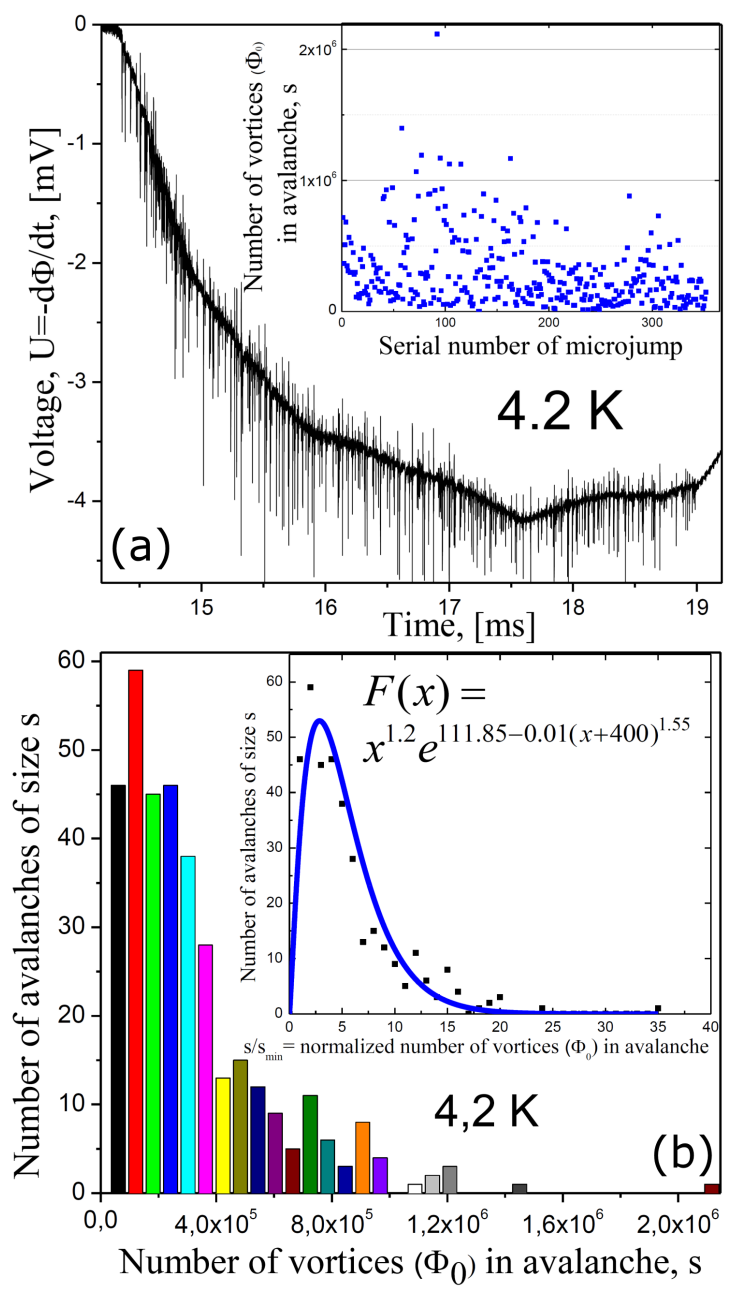

Fig. 3. (a) Main frame: fine structure of avalanche at $T=4.2 \mathrm{~K}$; inset: the magnitude of magnetic flux in microavalanches, depending on their serial number; (b) the distribution of microavalanches by size. Inset: the approximation of distribution function by analytical expression shows in figure.

Details of the structure of the outgoing avalanche observed in the fourth quadrant are shown in Fig. 3a, which clearly shows that the main impulse corresponding to macroavalanche is superimposed by small impulses interpreted as microavalanches. Time integration of the signal from the coil allows us to find the value of the entering (exiting) flux as a result of the avalanche at different temperatures. We suppose that individual microavalanches correspond to the exit of bundles of vortices that jump from individual pinning centers. Thus, calculating the area under the voltage pulse (per one turn of coil) equal to the flux of the microavalanche $\Delta \Phi_{\mu a v}$, we can determine the number of vortices $s$ trapped by individual pinning centers $\left(s=\Delta \Phi_{\mu a v} / \Phi_{0}\right.$, here $\Phi_{0}=$ $h / 2 e=2.067 \times 10^{-15} \mathrm{~Wb}-$ quantum of the flux $)$. The calculations performed show that each such bundle contains about $\left(10^{4} \div 10^{6}\right)$ vortices. The duration of individual microavalanches lies within range $5 \div 50 \mu \mathrm{s}$.
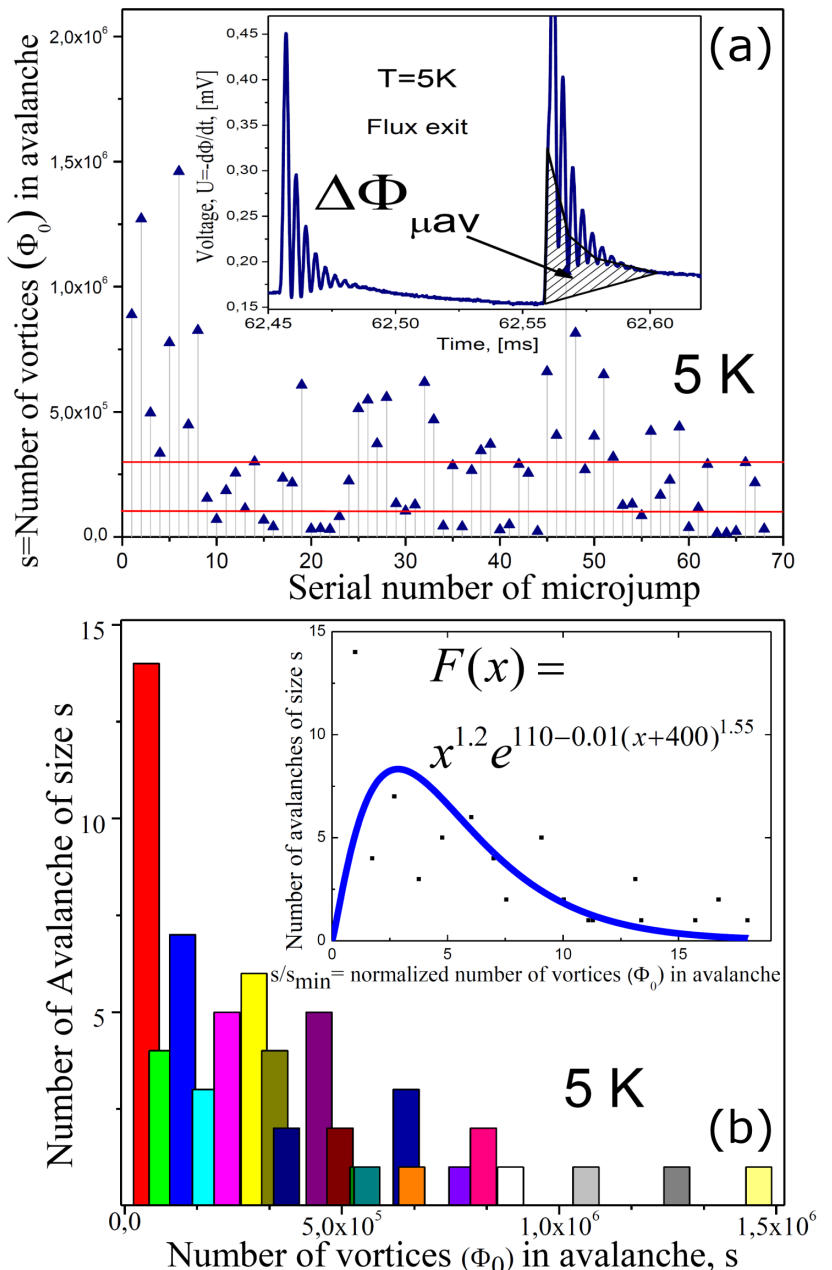

Fig. 4. The magnitude of magnetic flux in microavalanches, depending on their serial number at $T=$ $5 \mathrm{~K}$; inset: two solitary impulses of voltage in the fine structure are presented here; (b) the distribution of microavalanches by size. Inset: the approximation of distribution function by analytical expression shows in figure.

The inset in Fig. 3a shows the magnitudes of the magnetic flux in microavalanches which consistently leave the sample at $T=4.2 \mathrm{~K}$. An analogous distribution at $T=5 \mathrm{~K}$ is shown in Fig. 4a. Here, in the inset, the structure of pulses corresponding to individual microavalanches is shown. The area under the voltage pulse is equal to the value of flux in the microavalanche $\Delta \Phi_{\mu a v}$. The oscillations at the final stage of the flux jump have no physical meaning. They are due to the response of the resonance system (coil inductance and system capacity) 
to the flux pulse. Comparison of the data in Fig. 3 and Fig. 4 shows that as the temperature increases, both the number of vortices in microavalanches and their number decrease (almost threefold). This may be due to the fact that as the temperature increases, thermal energy $(E \sim k T)$ increases, which in turn leads to a decrease in the number of vortices bundle held by the pinning centers.

In Figs. 3b and 4b, histograms of the distribution of the number of microavalanches as a function of their sizes are shown for $4.2 \mathrm{~K}$ and $5 \mathrm{~K}$, respectively. The distributions have a characteristic form with a maximum near a certain value. The insets in Figs. $3 \mathrm{~b}$ and $4 \mathrm{~b}$ show the approximation of histograms by continuous curves. The histograms of the distributions at both temperatures are well approximated by the dependence with the maximum: $N(x)=x^{n} \exp \left(a-b(x+c)^{k}\right)$, here $N$ is the number of avalanches of size $s, x=s / s_{\min }$ is the avalanche size reduced to the minimal avalanche size. The values of the parameters of the $N(x)$ dependence are shown in the figures. The values of $n$ and $k$ for both temperatures coincide, this may indicate that the nature of the pinning centers does not change with a change in temperature.

Based on the number of vortices in microavalanches, one can roughly estimate the size of the crystal regions in which they are pinned. If we assume that the distance between the vortices at the pinning center is the same as in the triangular lattice, then the observed bundle with $10^{6}$ vortex should occupy an area of about $50 \mu \mathrm{m}^{2}$. It should be noted that this is not the size of the pinning center, since the vortices held by the pinning center occupy a larger area than the size of the center itself. It can be assumed that the capacity of the pinning center (the property of retaining (pinning) a certain number of vortices) is determined by its nature and size. In this case, the distribution of the number of microavalanches, as a function of the magnitude of the flux in them, can give information both about the distribution of the pinning centers in size $(s)$ and about their nature.

Unfortunately, it was not possible to answer definitely the question of the nature of the pinning centers in our sample. According to the value of the resistance ratio $\left(R_{300 \mathrm{~K}} / R_{20 \mathrm{~K}} \approx 12\right)$ we have that the mean free path is estimated to be as long as the Ginzburg-Landau coherence length $l \approx \xi_{G-L}[14]$ that is, our sample is in the middle between the limits of pure $l \gg \xi$ and a dirty superconductor $l \ll \xi$. The investigated crystal is quite perfect from the crystallographic point of view, which was confirmed by the X-ray studies. From inductive measurements, the width of the superconducting transition is only $0.27 \mathrm{~K}$ (Fig. 1b), which indicates that there are no significant amounts of ordinary defects (impurities, vacancies, etc.) that are intrinsic pinning centers. Assuming that there are intrinsic pinning centers, the scale of the defects $\left\langle d_{p c}\right\rangle$ should be of the order of the coherence length $\xi_{\mathrm{G}-\mathrm{L}}$. The estimate of the coherence length for $\mathrm{V}_{3} \mathrm{Si}$ crystal gives the value $\left(\xi_{\mathrm{G}-\mathrm{L}}\right)_{V 3 S i}=\left(\Phi_{0} / 2 \pi B_{c 2}\right)^{1 / 2} \approx 37 \AA$. This value is much smaller than the estimate of the size of the localization region of the vortex bundles obtained from the fine structure of the avalanche. At the same time, the structure of the transition line (Fig. 1b) indicates the presence of two phases in the sample with very close critical temperatures. It is difficult to say anything about the phases themselves, but the interphase boundaries can serve as flux pinning centers. Therefore, in the $A-15$ compounds, grain boundaries are empirically found to be especially strong pinning centers [15-18]. Another, larger scale of pinning centers could occur in the presence of residues of the cubic phase of the crystal after a structural phase transition from the cubic phase to the tetragonal phase at $T_{p t}=20.5 \mathrm{~K}[19]$. At the same time, it is unlikely that at a sufficiently low temperature $(4.2 \mathrm{~K})$ a sufficient number of domains of the cubic phase will be found to realize effective pinning.

We analyzed the dynamics of flux at avalanche in the framework of the magnetic flux diffusion model. The magnetic flux dynamics at the avalanche leads to a voltage pulse on the pick-up coil. This voltage varies according to law $U_{\text {coil }}(t) \sim \exp \left[\left(-D_{m} \pi^{2} t\right) /\left(4 d^{2}\right)\right]$, here $D_{m}$ is diffusion coefficient, $d$ is the characteristic size of the flux penetration region. The process of increasing the voltage to a maximum characterizes the actual development of the avalanche. At the second stage, when the flux front stopped, the incoming flux is redistributed in the sample as a result of a decrease in the temperature gradient.

At the top of Fig. 5 the signal from the coil is shown at $=4.2 \mathrm{~K}$ and $=5 \mathrm{~K}$. By building a voltage pulse on a semilogarithmic scale, it is easy to estimate the diffusion coefficient $D_{m}=4 d^{2} / \pi^{2} \tau_{0}$. In the process of avalanche development, a certain amount of heat is locally released in the sample and temperature gradients appear. Under such conditions, the relaxation times will be determined by the ratio of the coefficients of thermal and magnetic diffusion. Under the experimental conditions, it can be assumed that the relaxation time of the signal on the coil is determined by the diffusion of the magnetic flux $\left(D_{t h} \ll D_{m}\right)[1]$.

For the penetration depth of the avalanche flux front, we can take, approximately, one-half the diameter of the disc: $d=4.25 \times 10^{-3} \mathrm{~m}$. The basis for choosing such a value of the penetration front depth is the size of the area of the sample, from which an avalanche flux jumped out. The area can be estimated as follows. For example, for the jump observed in the second quadrant (inset in Fig. 2), the area under the curve $U(t)$ gives the measured value of the outgoing magnetic flux $\Delta \Phi=19.8 \mu \mathrm{Wb}$. The magnitude of the induction of the magnetic field in the sample before the avalanche, as measured by the Hall sensor, is $B_{\text {surf }}=\mu_{0} M+B_{\text {jump }}=0.59 \mathrm{~T}$ (hysteresis loop in Fig. 2). The ratio $\Delta \Phi / B_{\text {surf }}$ defines the area occupied by the avalanche, which in this case is $60 \%$ of the sample area. 

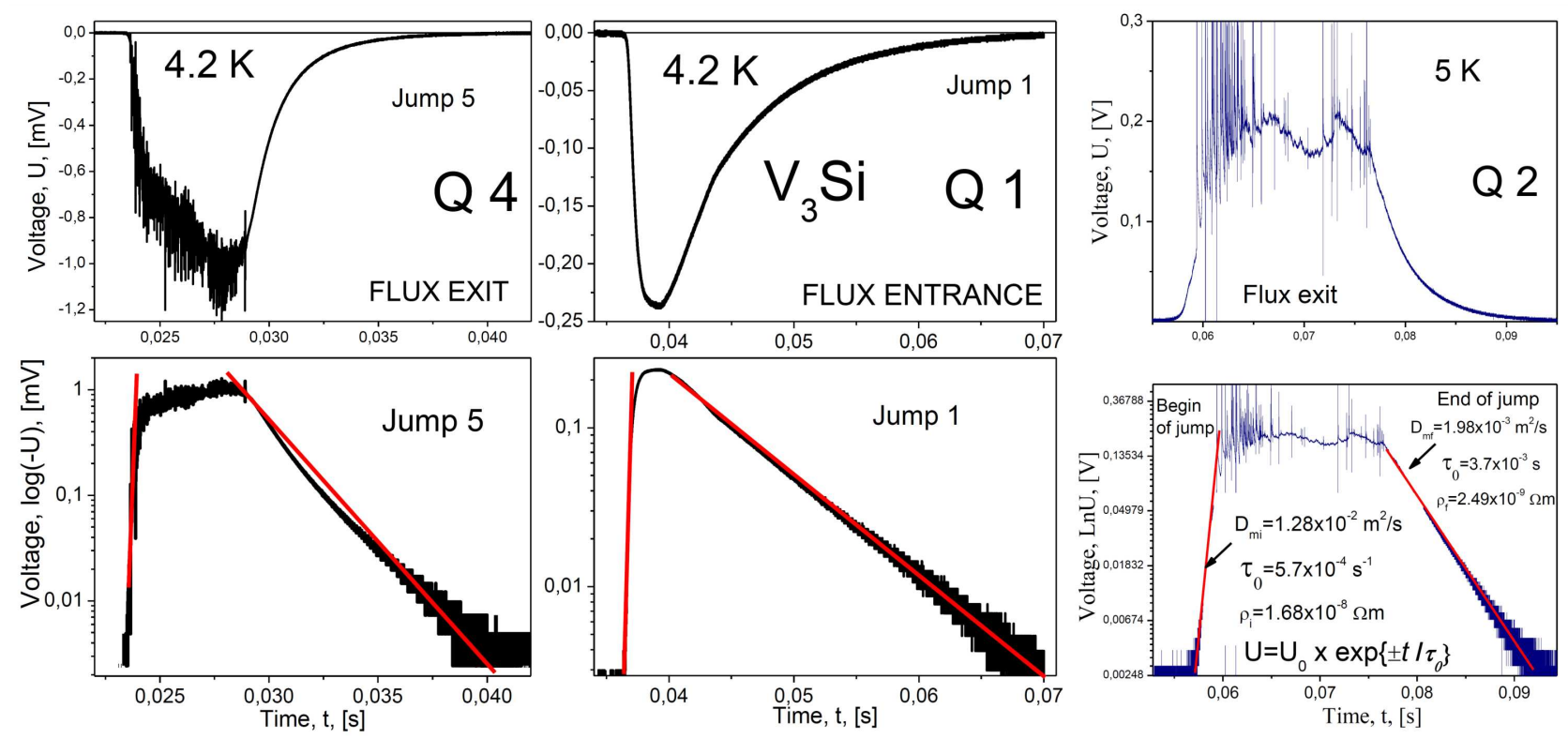

Fig. 5. Upper parts: voltages on the coils demonstrated the structure of avalanches at the magnetic flux entrance (Jump 1) and exit (Jump 5) at $T=4.2 \mathrm{~K}$ and $5 \mathrm{~K}$. Lower parts: logarithm of voltages for estimate of magnetic diffusivity at the beginning $\left(D_{m i}\right)$ and at the end $\left(D_{m f}\right)$ of the avalanche, $\tau_{0}$ is the duration of process, $\rho$ is the resistivity.

From the experimental data in Fig. 5 we determine the time of magnetic flux diffusion at the initial part of avalanche (the rise time of the signal $\tau_{i 0}$ ) and at the final part of process (the decay time of the signal $\tau_{f 0}$ ): $\tau_{i 0} \approx 5.7 \times 10^{-4} \mathrm{~s}, \tau_{f 0} \approx 3.7 \times 10^{-3} \mathrm{~s}$. Taking into account these values, for the corresponding diffusion coefficients we obtain: $D_{m i} \approx 1.28 \times 10^{-2} \mathrm{~m}^{2} / \mathrm{s}$ in the process of avalanche development and $D_{m f} \approx 1.98 \times 10^{-3} \mathrm{~m}^{2} / \mathrm{s}$ at the stage of relaxation (Fig. 5). As can be seen from these data at the final stage of the avalanche the diffusion coefficient decreases by more than an order of magnitude. This may be due to the fact that at the final stage the dynamics of the magnetic flux is largely determined by the rate at which the temperature equilibrium of the sample with the surrounding medium is established. The last one is determined by the rate of thermal diffusion, which occurs much more slowly than the magnetic one in local adiabatic condition. The resistivity in the flux move regime $\left(\rho=\mu_{0} D_{m}\right)$ in the corresponding periods of development of instability is about: $\rho_{i} \approx 1.68 \times 10^{-8} \Omega \mathrm{m}$ and $\rho_{f} \approx 2.49 \times 10^{-9} \Omega \mathrm{m}$ at the initial and final stage, respectively. This estimation allows us to conclude that the sample does not turn into a normal state, i.e. the heat generated is not sufficient to raise the temperature above the critical point.

\section{Conclusions}

- Fine structure of thermomagnetic avalanches outgoing from $\mathrm{V}_{3} \mathrm{Si}$ single crystal consists of several hundreds of microavalanches. The magnitude of the flux of the microavalanches and its duration were determined.
- The histograms of the sizes distribution of microavalanches have been plotted for $4.2 \mathrm{~K}$ and $5 \mathrm{~K}$ and found to approximate the form $N=x^{n} \exp (a-$ $\left.b(x+c)^{k}\right)$, here $N$ is number of microavalanches with size $s, x=s / s_{\min }$ is avalanche size, reduced to the minimal size. The values of $n$ and $k$ for both temperatures are identical. This may indicate that the nature of the pinning centers does not change in this temperature range.

- The dynamic of the flux jumps has been analyzed in the framework of the model of magnetic diffusion. The magnetic diffusivity at the initial stage $D_{m i} \approx 1.3 \times 10^{-2} \mathrm{~m}^{2} / \mathrm{s}$ of the thermomagnetic avalanche and at the final stage one decreases to about $2 \times 10^{-3} \mathrm{~m}^{2} / \mathrm{s}$. This value of the magnetic diffusivity is lower than that typical for the normal state. It means that maximal temperature of the sample during the thermomagnetic avalanche is lower than $T_{c}$.

\section{References}

[1] R.G. Mints, A.L. Rakhmanov, Rev. Mod. Phys. 53, 551 (1981).

[2] V. Chabanenko, B.N. Kodess, A. Nabiałek, E.I. Kuchuk, H. Szymczak, Phys. Proced. 36, 634 (2012).

[3] P. England, A. Inam, X.D. Wu, M.S. Hegde, B. Dutta, T. Venkatesan, Phys. Rev. B 41, 4834 (1990).

[4] S.T. Stoddart, S.J. Bending, R.E. Somekh, M. Henini, Supercond. Sci. Technol. 8, 459 (1995).

[5] V. Jeudy, D. Limagne, Phys. Rev. B 60, 9720 (1999). 
[6] T. Matsushita, Adv. Supercond. 6, 503 (1994).

[7] S. Field, J. Witt, F. Nori, X. Ling, Phys. Rev. Lett. 74, 1206 (1995).

[8] R.J. Zieve, T.F. Rosenbaum, H.M. Jaeger, G.T. Seidler, G.W. Crabtree, U. Welp, Phys. Rev. B 53, 11849 (1996).

[9] C. Heiden, G.I. Rochlin, Phys. Rev. Lett. 21, 691 (1968).

[10] E. Altshuler, T.H. Johansen, Rev. Mod. Phys. 76, 471 (2004).

[11] A. Nabiałek, A. Wiśniewski, V.V. Chabanenko, S. Vasiliev, F. Perez-Rodriguez, Supercond. Sci. Technol. 25, 035005 (2012).

[12] A. Nabiałek, S.V. Vasiliev, V.V. Chabanenko, X. Yao, Y.Q. Cai, L. Guo, M.V. Kuzovui, Acta Phys. Pol. A 121, 836 (2012).
[13] A. Nabiałek, V.V. Chabanenko, H. Szymczak, B.N. Kodess, J. Appl. Phys. 105, 063918 (2009).

[14] Y. Muto, N. Toyota, K. Noto, K. Akutsu, M. Isino, T. Fukase, J. Low Temp. Phys. 34, 617 (1979).

[15] K. Tachikawa, Y. Tanaka, Y. Iwasa, J. Appl. Phys. 44, 898 (1973).

[16] M. Suenaga, M. Garber, Science 183, 53 (1974).

[17] R.M. Scanlan, W.A. Fietz, E.F. Koch, J. Appl. Phys. 46, 2244 (1975).

[18] E.J. Kramer, G.S. Knapp, J. Appl. Phys. 46, 4595 (1975).

[19] L.R. Testardi, Rev. Mod. Phys. 47, 638 (1975). 